\title{
Iron Yoke Eddy Current Induced Losses with Application to The ALS Septum Magnets*
}

\author{
R. D. Schlueter
}

Advanced Light Source

Accelerator and Fusion Research Division

Lawrence Berkeley Laboratory

University of California

Berkeley, CA 94720

August 16, 1991

"This work was supported by the Director, Office of Energy Research, Office of Basic Energy Sciences, Materials Sciences Division of the U.S. Department of Energy, under Contract No. DE-AC03-76SF00098 


\title{
Iron Yoke Eddy Current Induced Losses with Application to the ALS Septum Magnets
}

\author{
Ross D. Schlueter
}

August 16, 1991

\begin{abstract}
The theoretical development of relations governing the eddy current induced losses in iron electromagnet yokes is reviewed. A baseline laminated electromagnet design is analyzed and a parametric study illustrates the sensitivity of core losses to perturbations of various geometrical, material, and excitation parameters. Core losses and field gradients for the ALS septum magnets are calculated. Design modifications capable of eliminating transverse and longitudinal field gradients are discussed.
\end{abstract}

\section{Summary}

For large enough $\tau \omega$, where $\tau \equiv \sigma \mu_{0} \mu_{r} d^{2}, \omega \equiv 2 \pi f$, and $f=$ excitation frequency, the B-field just inside the face of the iron yoke (or lamination) can be several times the value of that for an identical magnet having a DC excitation of equivalent magnitude. For a real material with nonlinear $B-H$ the ratio of $H$ just inside the iron face where $x=d$ to $H$ of the $D C$ case, $H\left(B_{\text {iron }}(d)\right) / H\left(B_{\text {iron } D C}\right)$ is several times the ratio of the corresponding $B_{\text {iron }}(d) / B_{\text {iron } D C}$, and thus losses in the iron can be huge. A subtle damping/wave propagation/saturation effect diminishes these losses somewhat in such a nonlinear material by limiting the maximum attained value of $B_{\text {iron }}(d)$.

For the ALS septum magnet iron losses are expected to be $\sim 1.40 \%$ and $\sim 1.05 \%$, at the entrance and exit, respectively. (A $1 \%$ loss corresponds to a $100 \mathrm{G}$ reduction of $B_{\text {gap }}$ for the $10 \mathrm{kG}$ excitation). The longitudinal field drop is thus expected to be $\sim 35$ $\mathrm{G}(0.35 \%)$. If desired, this gradient could be eliminated by tapering the gap $\sim+0.35 \%$ ( $\sim 0.002$ inches) from entrance to exit.

The transverse field drop is expected to be $\sim 20 \mathrm{G}(0.20 \%)$. If desired, this gradient could be eliminated by slotting the laminations at the location of the coil/gap junction.

\section{Theoretical Groundwork}

Here we investigate the eddy-current induced losses in a solid core or laminated elctromagnet, following the theoretical development by Halbach ${ }^{[1,2]}$. 


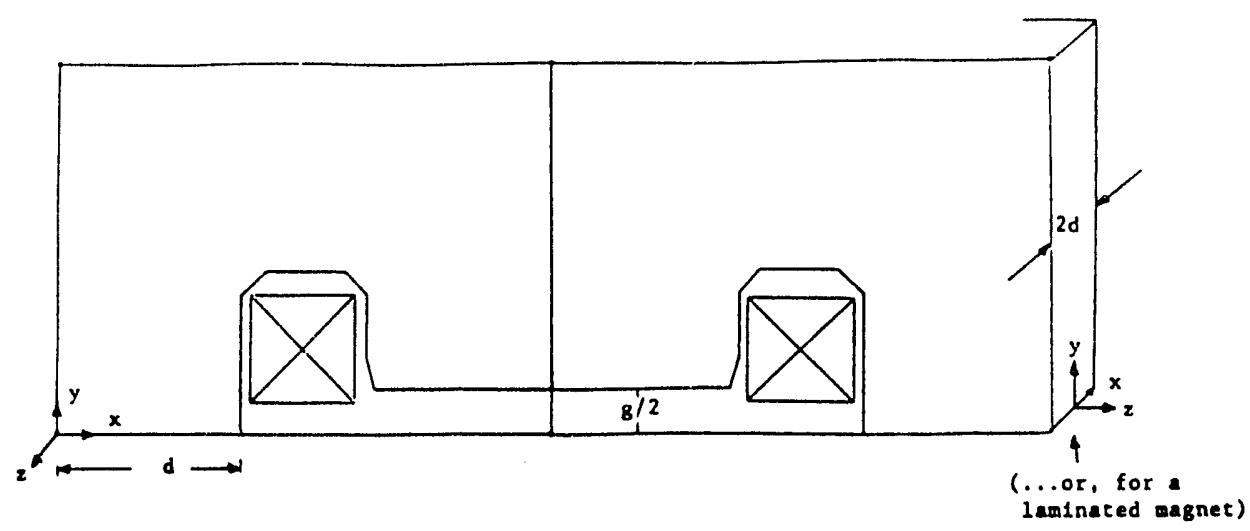

Figure 1. Generic upper-half electromagnet cross section

Assume that to first order in the region of interest in Figure 1 the variables are invariant with $y$ and $z, B=B_{y}(x), H=H_{y}(x), E=E_{z}(x), A=A_{z}(x) !^{\prime} \equiv \frac{\partial}{\partial x}$ and that ${ }^{\cdot} \equiv \frac{\partial}{\partial t}$. Ampere's Law for the circuit across the gap and just inside the iron face (or any path just inside the face of a lamination) is

$$
\mu_{0} I=\mu_{0} \oint H \cdot d s=g B_{\text {gap }}\left(1+\frac{L}{g} \frac{H_{\text {iron }}(d)}{H_{\text {gap }}}\right)=g B_{\text {gap }}\left(1+\frac{L}{g} \frac{B_{\text {iron }}(d)}{B_{\text {gap }}} \frac{1}{\mu_{r}}\right)
$$

where $L$ is the effective path length in the iron and $\mu_{r}=\mu_{r}\left(B_{\text {iron }}(d)\right)$. Assuming losses in the linearly behaved iron are small, a coil excitation $I(t)$ gives rise to a field in the gap $B_{\text {gap }}(t) \propto I(t)$ and

$$
\bar{B}_{\text {iron }}(t)=B_{\text {gap }}(t) \frac{w_{\text {gap }}}{w_{\text {iron }}}
$$

where the ' -' denotes the $x$-direction spatial average over $0-d$, with $d$ being the yoke width (or, for a laminated magnet, the lamination half-thickness, in which case we switch the coordinate axes $x \rightarrow-z, z \rightarrow x$ ).

From field equations $B=\nabla \times A$, and $\nabla \times E=-\dot{B}, \Longrightarrow E=-\dot{A}$. This result, combined with $\nabla \times H=J=\sigma E, \Longrightarrow H^{\prime}=\sigma \dot{A}$. The vector potential is thus given by

$$
\frac{\partial A}{\partial t}=\frac{1}{\sigma} \frac{\partial\left(\frac{1}{\mu} \frac{\partial A}{\partial x}\right)}{\partial x}
$$

subject to $A(x, 0)=$ given, $A(0, t)=0$, and $A(d, t)=-\bar{B}_{\text {iron }}(t) d$. Numerically, the following algorithm is convenient:

$$
A(x, t) \Longrightarrow B(x, t) \Longrightarrow H(x, t) \Longrightarrow \dot{A}(x, t) \Longrightarrow A(x, t+\delta t)
$$

Program septum_rk 4 in Appendix A uses a fourth order Runge Kutta algorithm to solve for the 1-D eddy current induced spatial field distribution in a yoke (or lamination) at chosen time intervals. Program sep_edge_rk \& in Appendix $A$ similarly solves for the temporal behavior of various parmeters of interest ircluding $B_{\text {iron }}(d, t), H\left(B_{\text {iron }}(d, t)\right)$, $\bar{B}_{\text {iron }}(t)$, and $H\left(\bar{B}_{\text {iron }}(t)\right)$. 

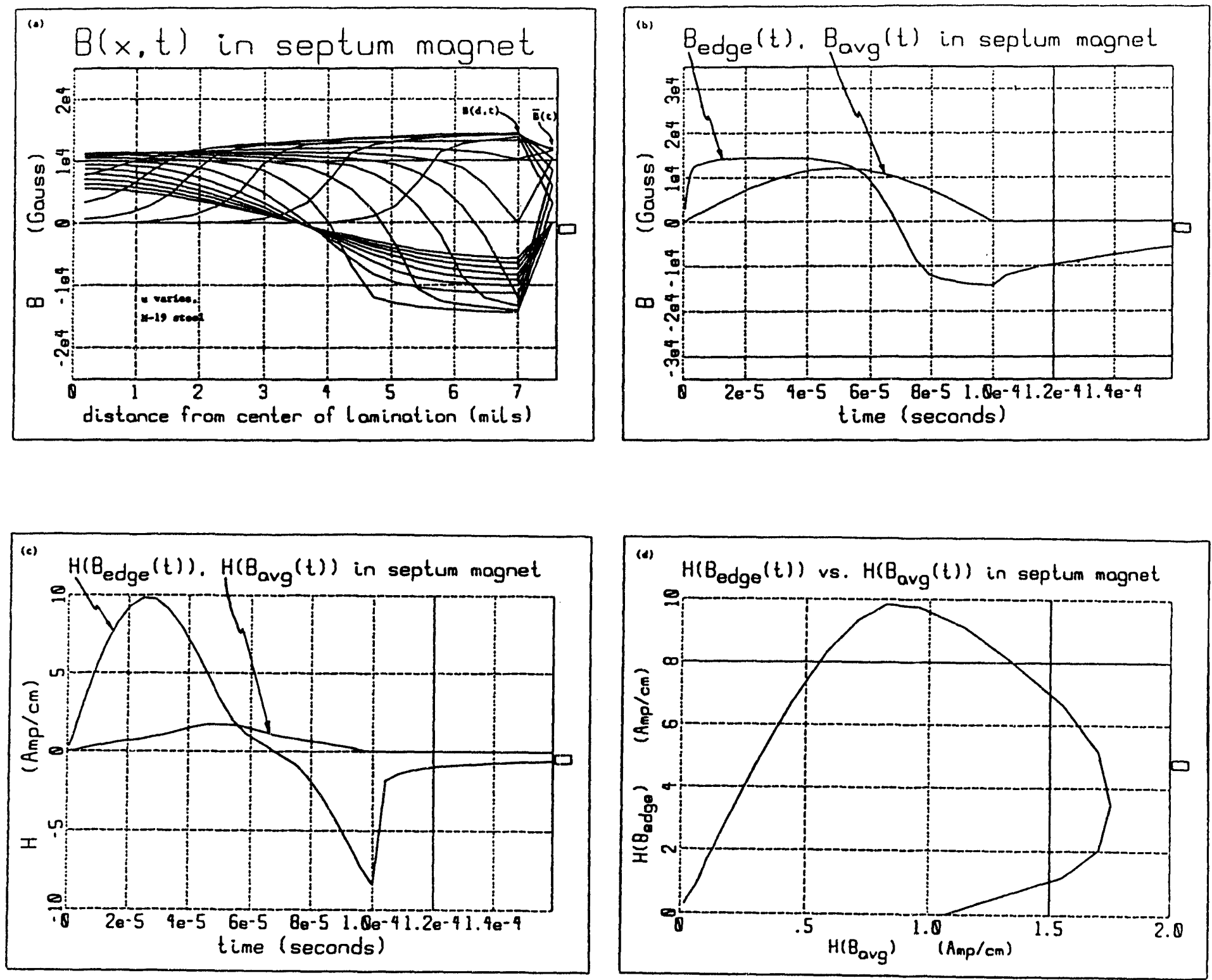

$$
\begin{aligned}
& \bar{B}_{\text {iron }}(t)=-A(d) / d=B_{0} \sin w t, \quad 0 \leq t<\pi / w, \quad B_{0}=12 \mathrm{kG}, \\
& =0, \quad \pi / w \leq t
\end{aligned}
$$

$1 / \sigma=50 \mu \Omega-\mathrm{cm}, f=\omega / 2 \pi=5 \mathrm{kHz}, d=0.007$ inch, $\mu$ for $\mathrm{M}-19$ transformer steel

Figure 2a,b,c,d. $B$ and $H$ distributions and temporal history for baseline eddy current induced losses in a laminated magnet. 


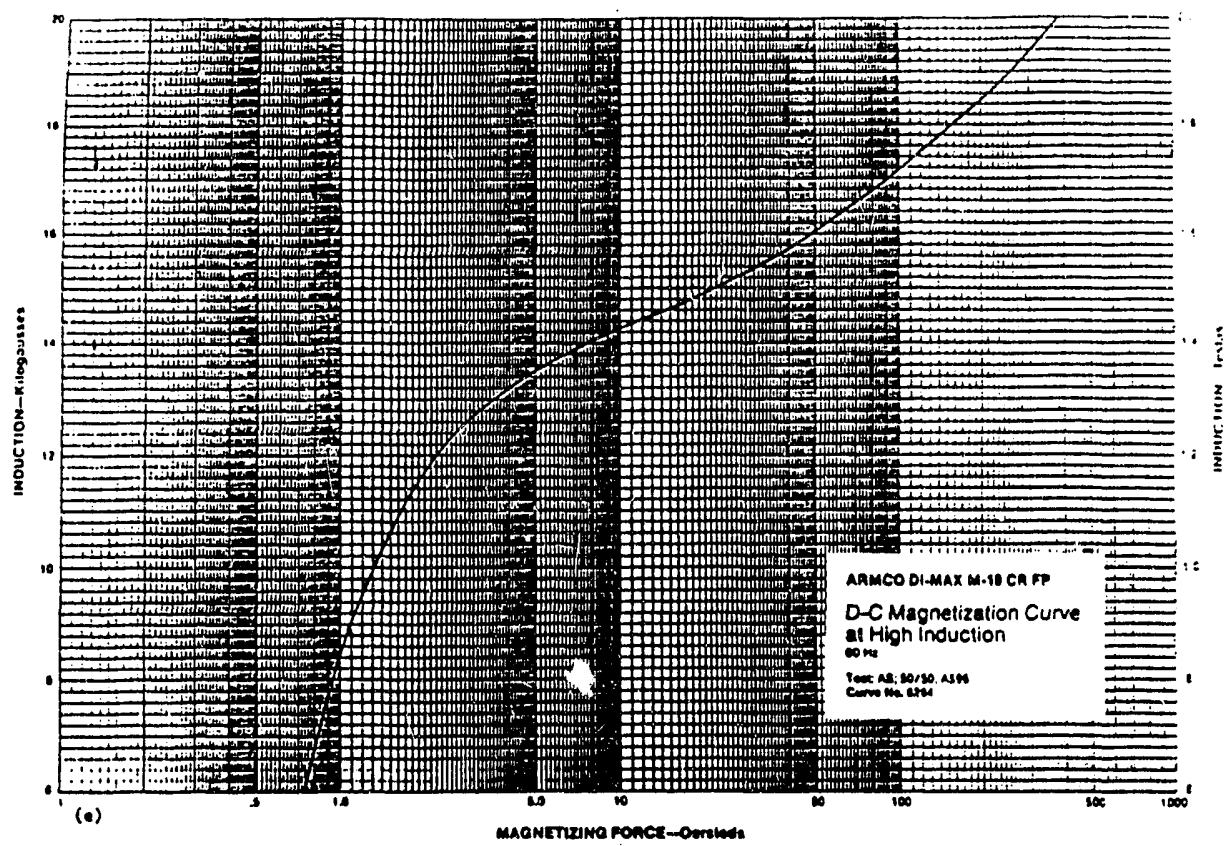

Figure 2e. B-H curve for M-19 transformer steel

\section{Baseline: An Eddy Current Induced Loss Calculation in a Laminated Magnet}

Assuming a gap excitation $B_{\text {gap }}(t)=B_{\text {gapmax }} \sin \omega t, \quad 0 \leq t<\pi / \omega$, then $\bar{B}_{\text {iron }}(t)=$

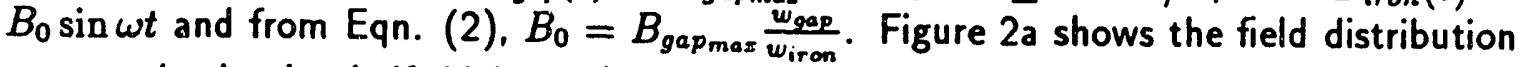
across a lamination half-thickness ( $0-0.007$ inches) at time intervals of $\frac{1}{12}$ of $100 \mu \mathrm{sec}$ for the baseline parameters given. Also shown on the right in the Figure are $\bar{B}_{\text {iron }}$ values corresponding to the field distribution at these time intervals. Figures $2 b$ and $2 c$ show the temporal $B$ and $H$ history, respectively, at the lamination face $(x=d)$ along with the average (excitation) values. The $H\left(B_{\text {iron }}(d, t)\right)$ and $H\left(\bar{B}_{\text {iron }}(t)\right)$ in Figure $2 d$ are proportional to, respectively, the eddy current induced losses and those of a DC excitation of the same magnitude. The B-H curve for M-19 transformer steel used is given in Figure $2 e$ and is listed in the programs in Appendix $A$.

From Eqn. (1), assuming $\frac{L}{g} \simeq 10$ and $\frac{w_{\text {gap }}}{w_{\text {iron }}} \simeq 1$, eddy current induced losses in the iron for this baseline case are $1.03 \%$. This is over five times the loss level of a DC excitation of the same magnitude.

\section{Parametric Study: Eddy Current Induced Losses in Lam- inated M-19 Transformer Steel and in Steel with $\mu_{r}=1000$}

Figure 3a lists data of a parametric study for perturbations about the baseline. The left third of the figure lists the varied paramters, the middle third lists maximum $B$ and $H$ in the iron along with losses in the M-19 transformer steel, and the right third lists data for the same parameters but for a fictitious constant permeability steel with $\mu_{r}=1000$. Also included in the right third are numerical data for a continuous sinusoidal excitation; these 
results agree with the closed form solution ${ }^{[2]}$ shown in Figure 3c. Here $\left.B_{\text {iron }}(d)\right|_{\max } / B_{0}=$ $\alpha / \tanh \alpha$, where $\alpha \equiv \sqrt{i \tau \omega}$. In Figure $3 b$ are plotted the excitation $H\left(\tilde{B}_{\text {iron }}(t)\right)$ and the response $H(B(d, t))$ histories to a half-sine wave excitation in $M-19$ transformer steel for the parameters listed in Figure 3a.

Effect of $\mu_{r}$. Application of the relation given in Figure $3 c$ shows that for large enough $\tau \omega, \overline{\left.B_{\text {iron }}(d)\right|_{\max }}$ can be several times $B_{0}$; for a real material with nonlinear $B-H$ the corresponding $H\left(\left.B_{\text {iron }}(d)\right|_{\max }\right) / H\left(B_{0}\right)$ is several times the $\left.B_{\text {iron }}(d)\right|_{\max } / B_{0}$ ratio, and thus losses in the iron can be huge. A subtle damping/wave propagation/saturation effect diminishes these losses somewhat in such a nonlinear material by limiting the maximum attained value of $\left.B_{\text {iron }}(d)\right|_{\text {max }}$.

\begin{tabular}{|c|c|c|c|c|c|c|c|c|c|c|}
\hline$\#$ & \multicolumn{4}{|c|}{ parameters } & \multicolumn{3}{|c|}{ M-19 transformer steel } & \multicolumn{3}{|c|}{$\mu_{r}=1000$} \\
\hline & $\begin{array}{l}B_{0} \\
k G\end{array}$ & $\begin{array}{c}1 / \sigma \\
\mu \Omega-\mathrm{cm}\end{array}$ & $\underset{k H z}{f}$ & $\underset{\text { mils }}{d}$ & $\begin{array}{c}B_{\text {iron }}(d) \\
\max . \\
k G\end{array}$ & $\begin{array}{l}H(d) \\
\max . \\
\mathrm{A} / \mathrm{cm}\end{array}$ & $\begin{array}{l}\max \text { loss in iron } \\
\frac{L}{g}=10, \frac{w_{\text {gap }}}{w_{\text {iron }}}=1 \\
\% \text { of excitation }\end{array}$ & $\tau \omega$ & $\begin{array}{c}B_{\text {iron }} \\
\text { half-sine } \\
\text { wave }\end{array}$ & $\begin{array}{l}\text { (d) }\left.\right|_{\text {max }} \\
\text { continuous } \\
\text { excitation }\end{array}$ \\
\hline $\begin{array}{l}0 \\
a \\
b \\
c \\
d \\
\text { e }\end{array}$ & $\begin{array}{l}12 \\
12 \\
14 \\
12 \\
12 \\
12\end{array}$ & $\begin{array}{l}50.0 \\
50.0 \\
50.0 \\
12.5 \\
50.0 \\
50.0\end{array}$ & $\begin{array}{c}D C \\
5 \\
5 \\
5 \\
20 \\
20\end{array}$ & $\begin{array}{c}7 \\
7 \\
7 \\
7 \\
7 \\
14\end{array}$ & $\begin{array}{l}12.00 \\
14.49 \\
14.71 \\
15.90 \\
15.90 \\
15.90\end{array}$ & $\begin{array}{l}1.75 \\
9.87 \\
13.2 \\
35.5 \\
35.5 \\
35.5\end{array}$ & $\begin{array}{l}.183 \\
1.03 \\
1.18 \\
3.73 \\
3.73 \\
3.73\end{array}$ & $\begin{array}{c}0 \\
2.5 \\
2.5 \\
10 . \\
10 . \\
10 .\end{array}$ & $\begin{array}{c}1.60 \\
16.47 \\
19.21 \\
31.87 \\
31.87 \\
31.87\end{array}$ & $\begin{array}{c}1.00 \\
16.62 \\
19.40 \\
37.70 \\
37.70 \\
37.70\end{array}$ \\
\hline
\end{tabular}
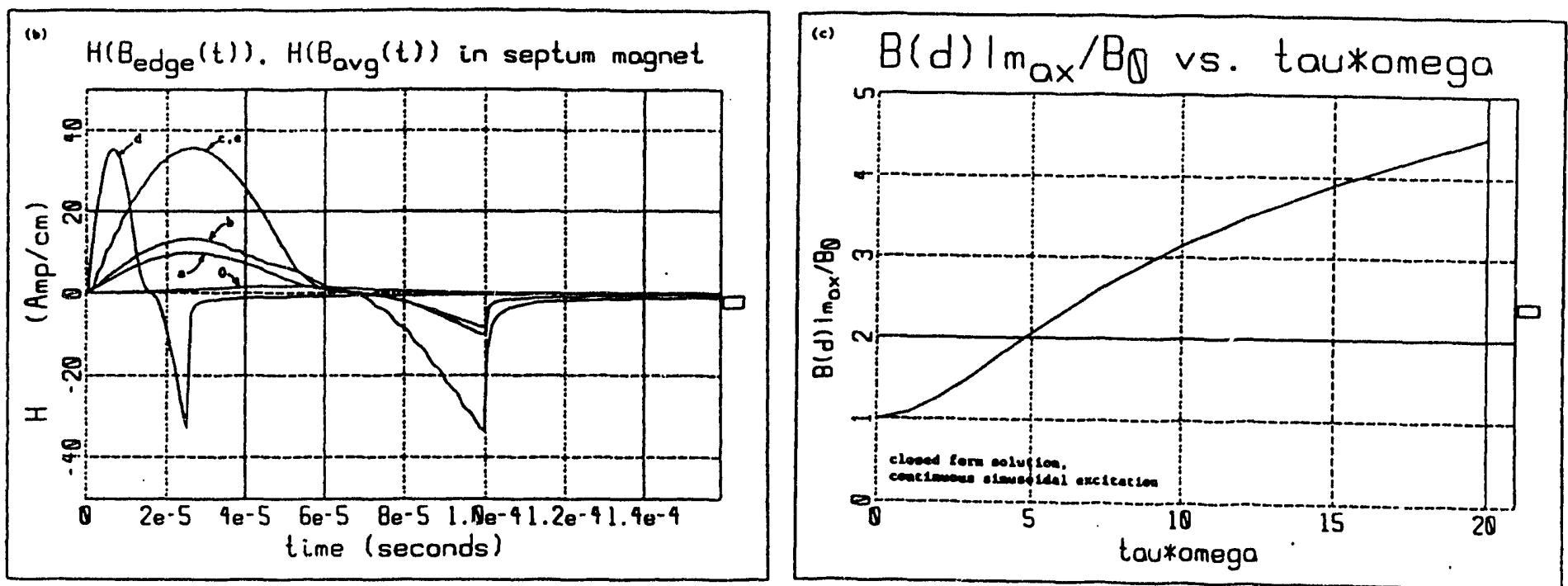

Figure $3 a, b, c$. Parametric study data of eddy current induced losses in a laminated magnet. 

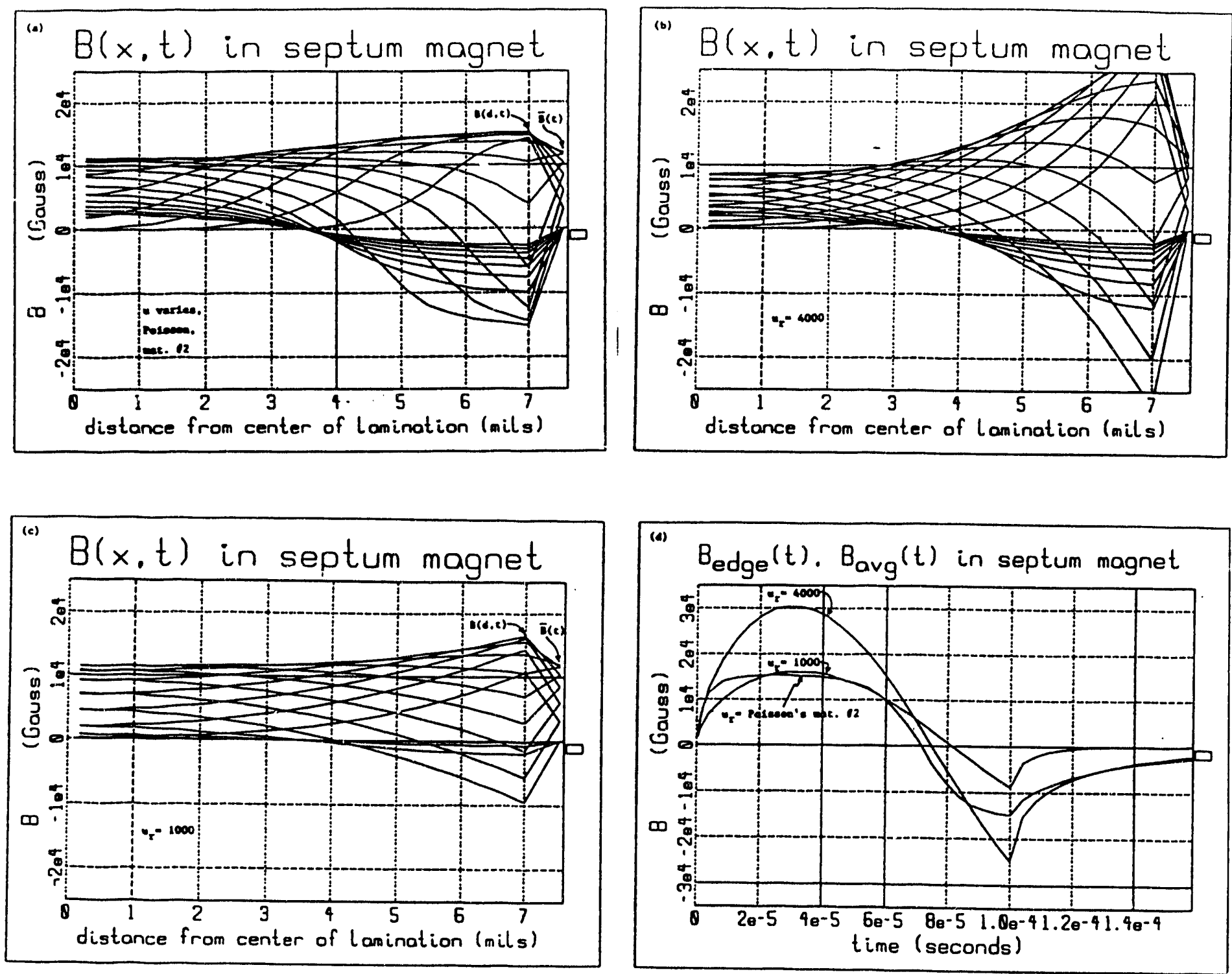

$$
\begin{aligned}
\bar{B}_{\text {iron }}(t)=-A(d) / d & =B_{0} \sin w t, \quad 0 \leq t<\pi / w, \quad B_{0}=12 \mathrm{kG}, \\
& =0, \quad \pi / w \leq t \\
1 / \sigma=50 \mu \Omega-\mathrm{cm}, \quad f & =\omega / 2 \pi=5 \mathrm{kHz}, \quad d=0.007 \text { inch }
\end{aligned}
$$

Figure $4 a, b, c, d$. Effect of iron permeability on eddy current induced losses in a laminated magnet. 
For the baseline parameters of Figure 2, Figures 4a,b,c show the B-field wave propagation in the half-lamination for (1) soft iron (Poisson default Material \#2) steel, (2) $\mu_{r}=1000$, and (3) $\mu_{r}=4000$, respectively. Figure $4 \mathrm{~d}$ shows temporal history of $B_{\text {iron }}(d, t)$ for the various cases in Figures $4 a, b, c$. Notice that for the nonlinear iron case the permeability ranges $1250<\mu_{r} \leq 4000$ everywhere and for all times, yet because of the damping/wave propagation/saturation interplay mentioned above, $\left.B_{\text {iron }}(d)\right|_{\max }$ never reaches the level of even that of the case with constant $\mu_{r}=1000$.

\section{Iron Losses in the ALS Septum Magnet}

\subsection{Iron Losses at the Entrance and Exit; Gap B-field Longitudinal Gra- dient}

Figures $5 \mathrm{a}, \mathrm{b}$ show cross sections of the ALS septum magnet entrance and exit where the gap width is $\sim 86 \%$ and $\sim 68 \%$ of the yoke width, respectively. Current strawman paramters for this magnet are: $B_{\text {gapmas }}=10 \mathrm{kG}, 1 / \sigma=50 \mu \Omega-\mathrm{cm}, f=5 \mathrm{kHz}$, and $M-19$ transformer steel lamination half thickness $d=0.007$ inches.

Figure 6a,b show eddy current induced loss tabulations from sep_edge_rk \& for three $\frac{w_{\text {gap }}}{w_{\text {iron }}}$ ratios. For this magnet the estimated $\frac{L}{g}=20$. Thus, a first approximation to the entrance and exit losses are $1.34 \%$ and $0.89 \%$, respectively. (A $1 \%$ losss corresponds to a $100 \mathrm{G}$ reduction in $\left.B_{\text {gap max }}\right)$. A [n improved?] calculation could account for an initial $w_{\text {wep }}$ ratio of $\sim 1$ near the gap/steel interface. Then $\int H \cdot d s_{i r o n} \simeq \Sigma_{i=1}^{2} H_{i} d s_{i}$. For the entrance $H_{1}=7 \mathrm{~A} / \mathrm{cm}, d s_{1}=6 \mathrm{gcm}, H_{2}=5.32, d s_{2}=14 \mathrm{~g}$, and losses come to $175 \mathrm{~A}$ $(1.45 \%)$. For the exit $H_{1}=7, d s_{1}=5 g, H_{2}=3.51, d s_{2}=17 g$, and losses come to 140 A $(1.19 \%)$.

If desired, this gradient could be eliminated by tapering the gap $\sim+0.35 \%(\sim+0.002$ inches) from entrance to exit to compensate for the decreasing losses in the iron as one travels from the magnet entrance to exit.

\subsection{Gap B-Field Transverse Gradient}

For $B_{g a p_{\max }}=10 \mathrm{kG}$, the field on the face of a lamination near the gap/iron interface can reach $\sim 14.2 \mathrm{kG}$ (see Figure 6a).

Poisson runs for the ALS septum magnet entrance and exit cross section geometries with the static $B_{\text {gap }}$ set to $14.2 \mathrm{kG}$ (so as to simulate the gap/iron eddy current interface conditon for a $5 \mathrm{kHz}$ half sine wave excitation of magnitude $10 \mathrm{kG}$ ) result in a transverse field gradients of $\sim 20 \mathrm{G}$ ( $\simeq 0.20 \%$ of the $10 \mathrm{kG}, 5 \mathrm{kHz}$ field in the gap).

The gradient arises because, although at all locations and times $\mu_{r} \gg 1$, it is not infinite. This gives rise to a nonzero $H_{/ /}$along the gap/iron interface in the transverse direction. Forcing $H$ to be perpendicular to this interface, for example by slotting the laminations in the axial direction at the location of the coil/gap junction would largely eliminate the transverse field gradient ${ }^{[3]}$. 

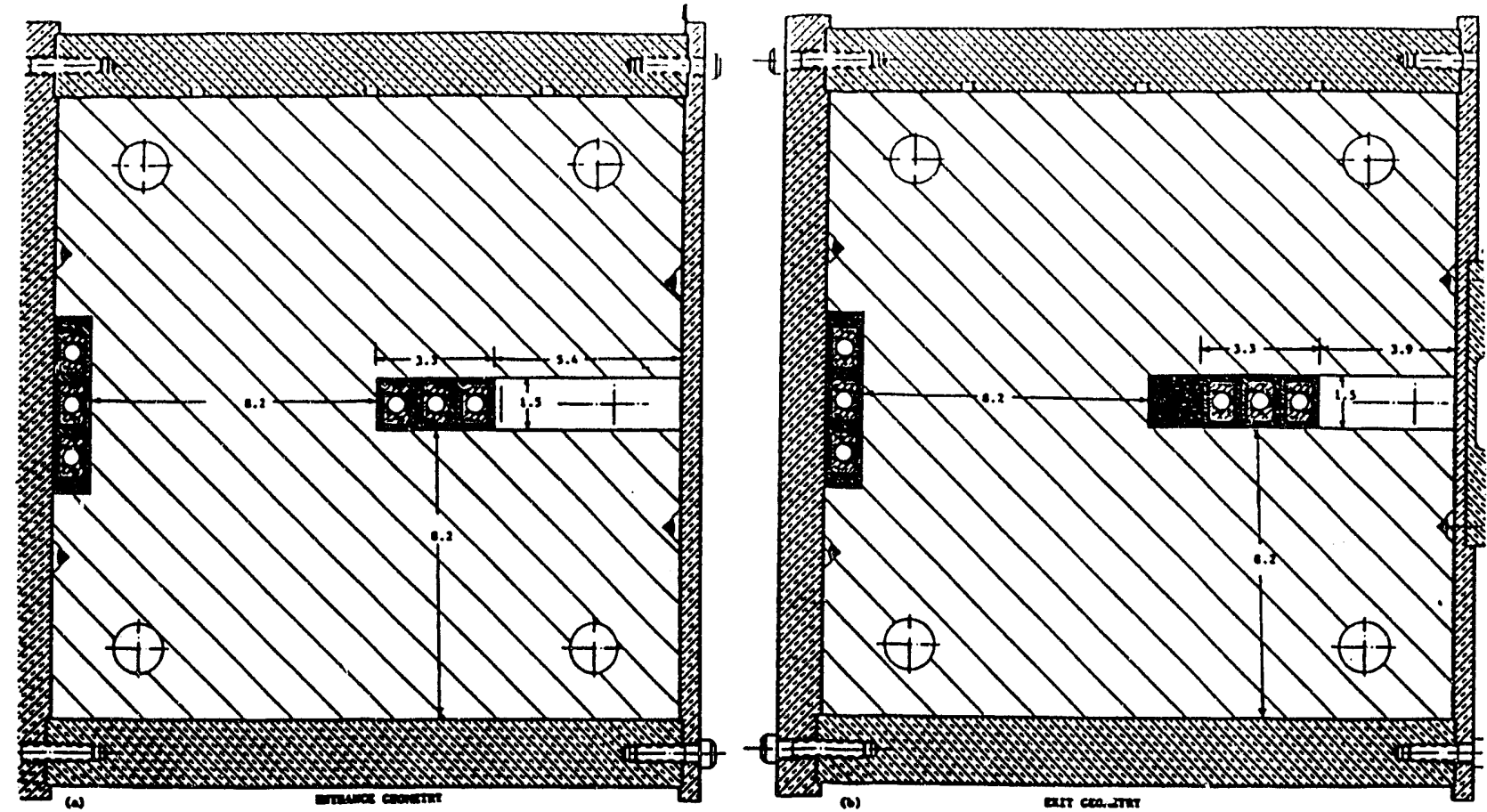

Figure 5. Cross sections of the ALS septum magnet entrance and exit

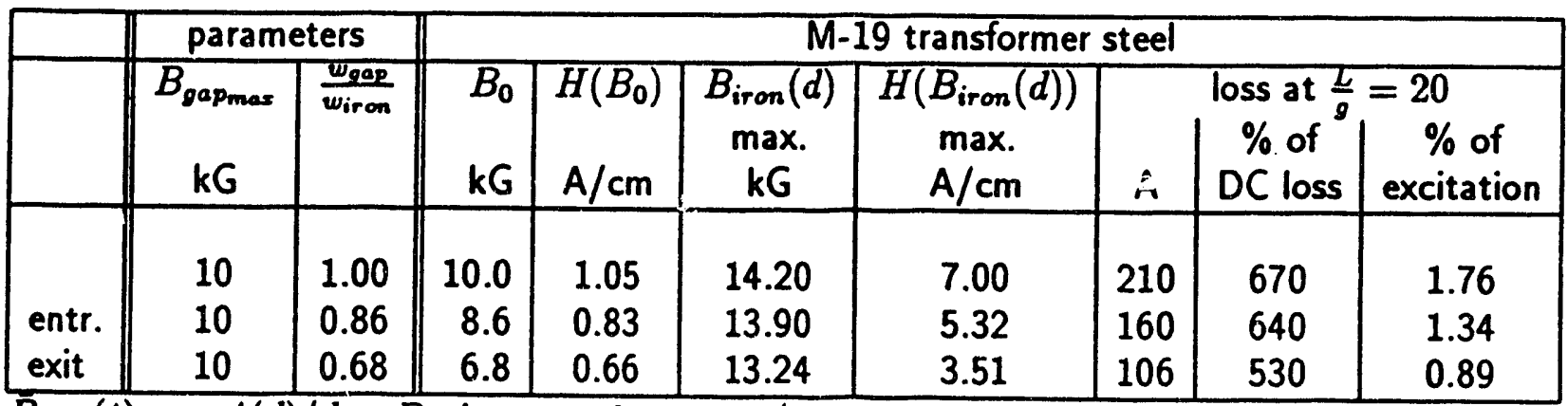

$B_{\text {iron }}(t)=-A(d) / d=B_{0} \sin w t, \quad 0 \leq t<\pi / w$

$$
=0 . \quad \pi / w \leq t
$$

$1 / \sigma=50 \mu \Omega-\mathrm{cm}, f=\omega / 2 \pi=5 \mathrm{kHz}, d=0.007$ inch, $\mu$ for $\mathrm{M}-19$ transformer steel

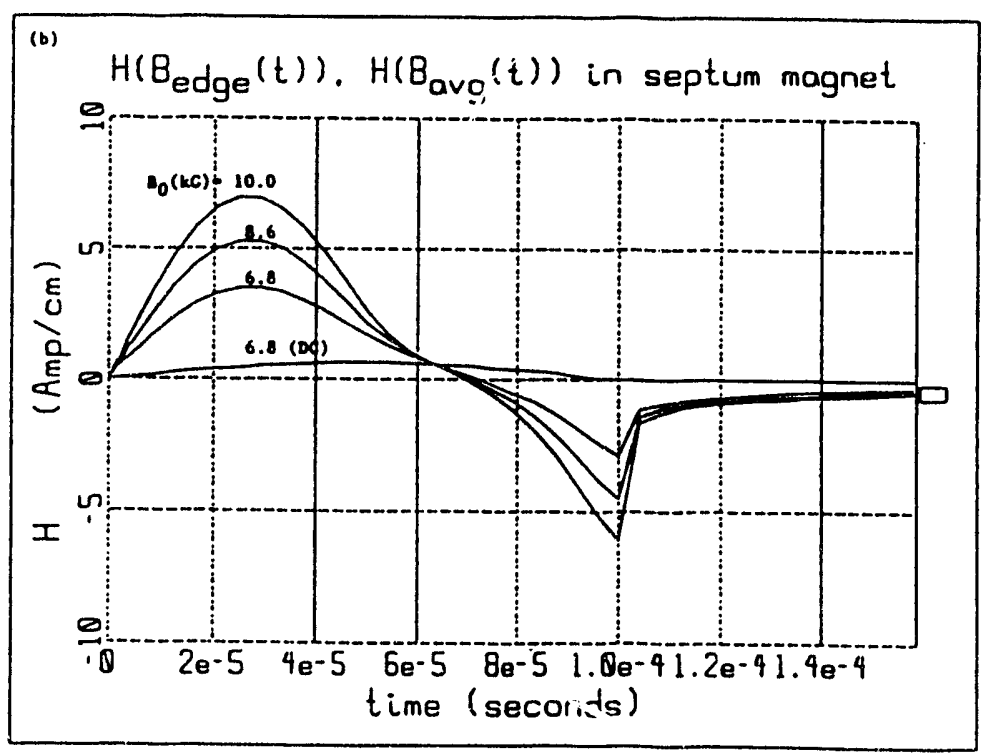

Figure 6a,b. Eddy cuirent induced losses in ALS septum magnet 


\section{Acknowledgements}

I am happy to acknowledge Klaus Halbach's guidance in the theoretical aspects of the work presented herein.

\section{Literature Cited}

1. Halbach, K., Some Eddy Current Effects in Solid Core Magnets, NIM 107, 529549, 1973.

2. Halbach, K., 1990 Magnet Technology Lectures at LBL, LBL Report V-902-2.1-6, pp. 1.4 - 1.11, April 1990.

3. Halbach, K., Jülich Magnet Lecture Series, pp 2.3 - 2.5, Jülich, Germany, 1985. 
Appendix A: Codes Septum_rk4 and sep_edge_rk4 


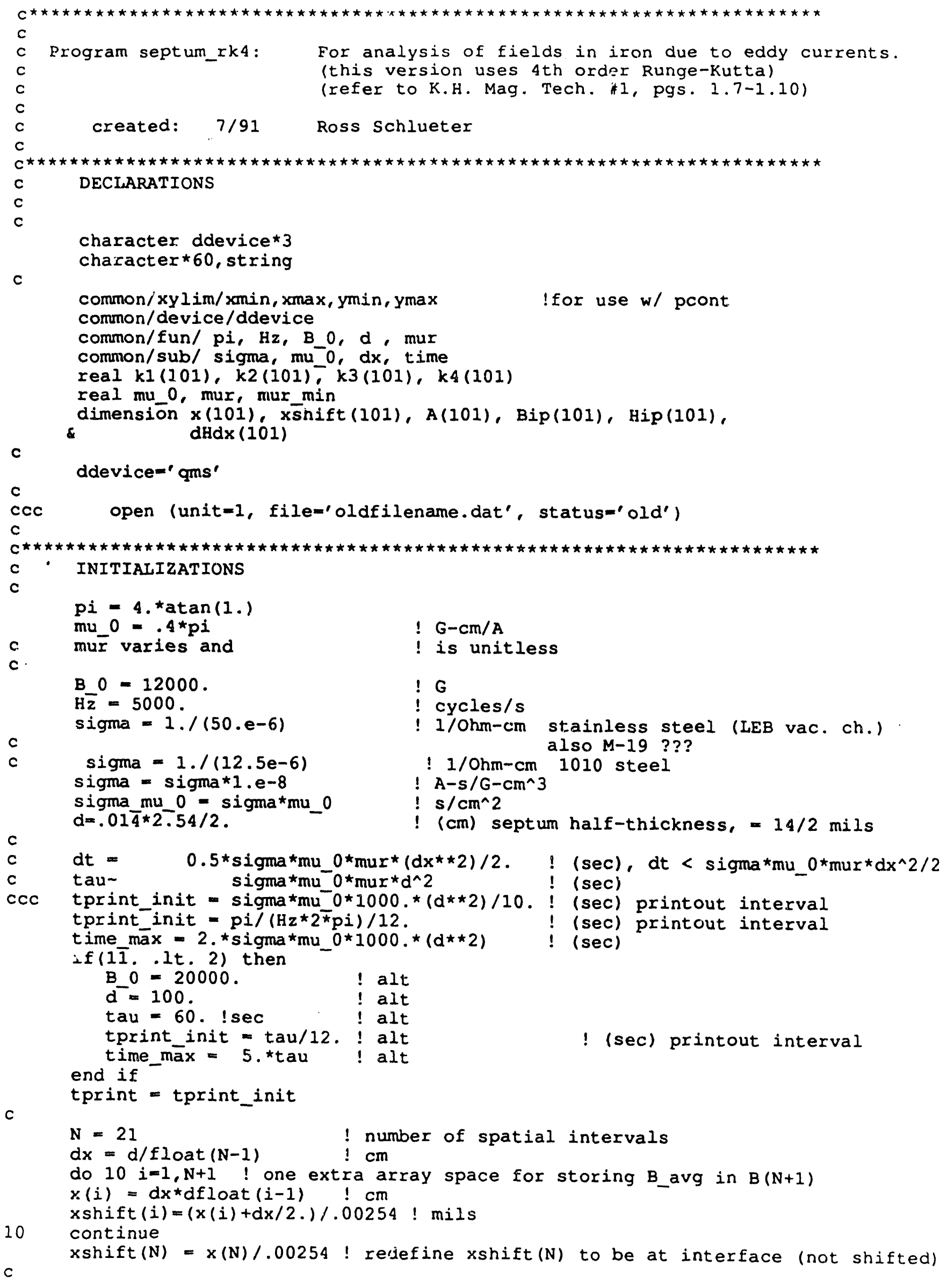




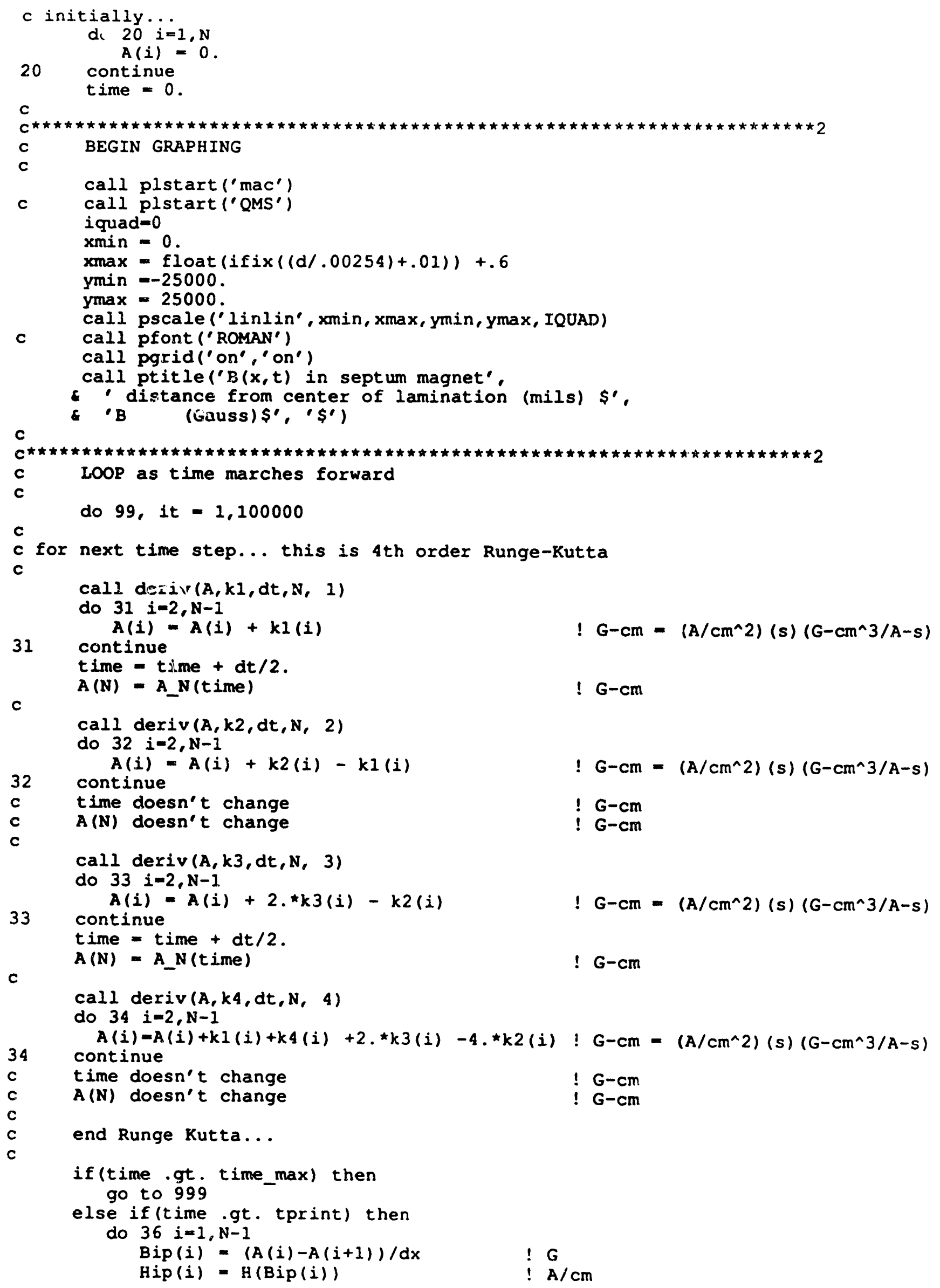




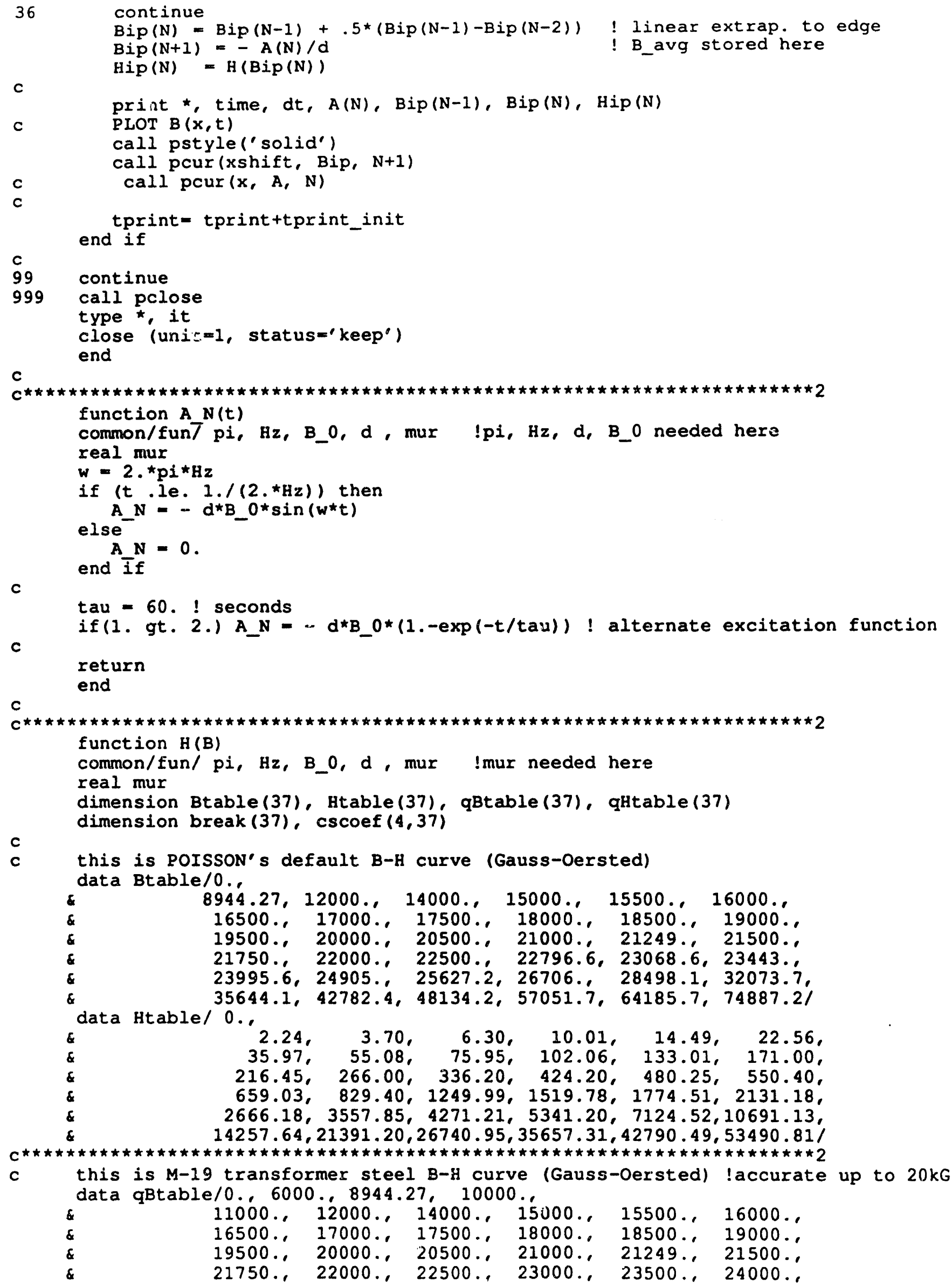




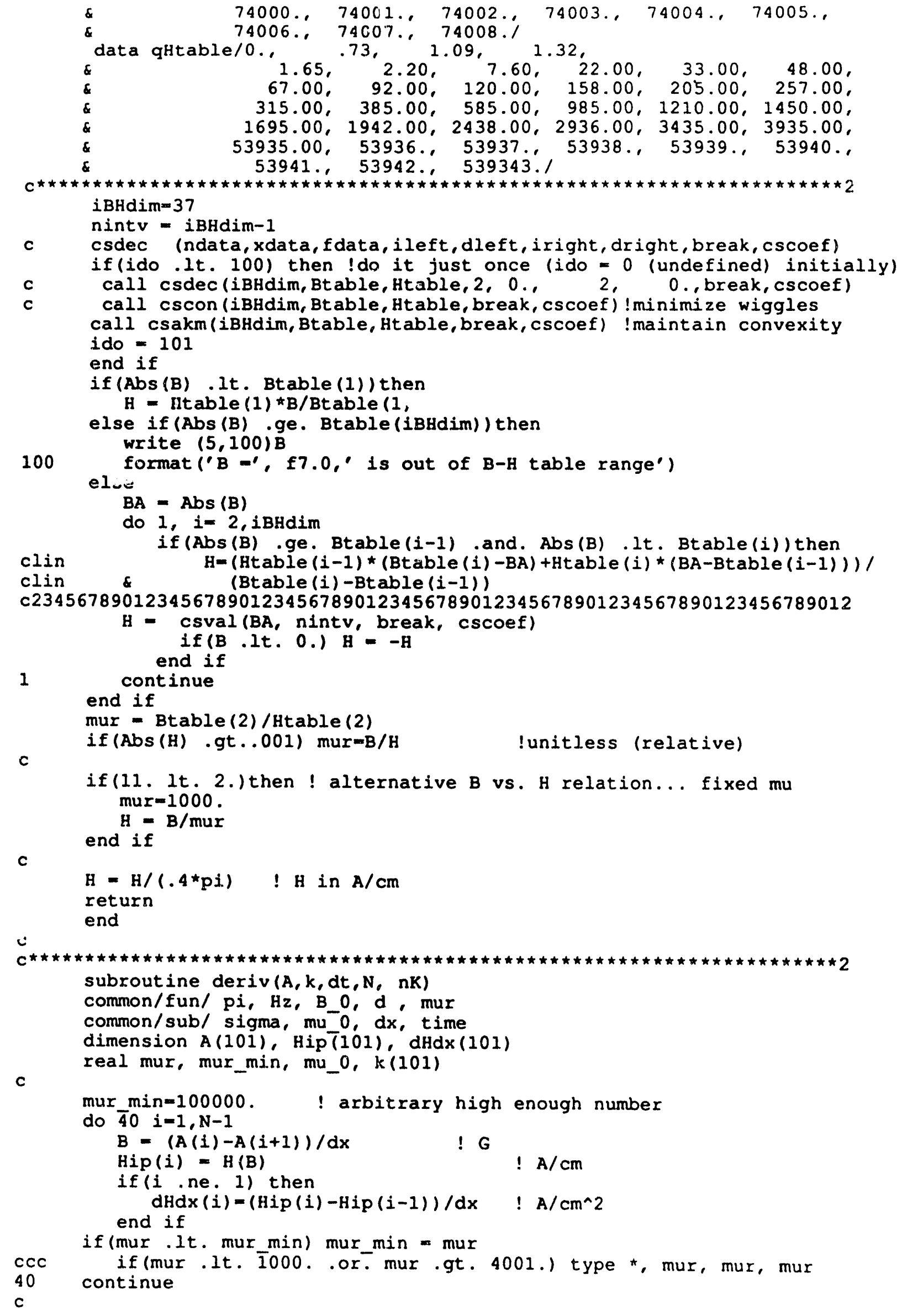


mur $=$ mur min

! $($ sec $), d t<$ sigma ${ }^{\star} m u \_0 * m u r \star d x^{\wedge} 2 / 2$

if $(n K . \bar{e} q .1) d t=.05 *$ sigma*mu $0{ }^{*} \operatorname{mur}{ }^{\star}\left(d x^{\star} \star 2\right) / 2$.

ccc if (nK.eq. 1) type *, dt, time, $A(N), B, H i p(N-1), n K$ do $41 i=1, N-1$

$k(i)=-d H d x(i) \star d t /\left(\right.$ sigma $\left.{ }^{2}.\right)$

41 continue

return

end

$c$

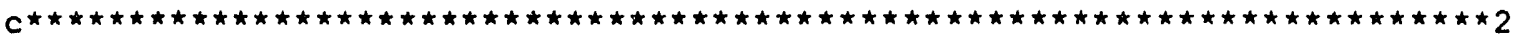




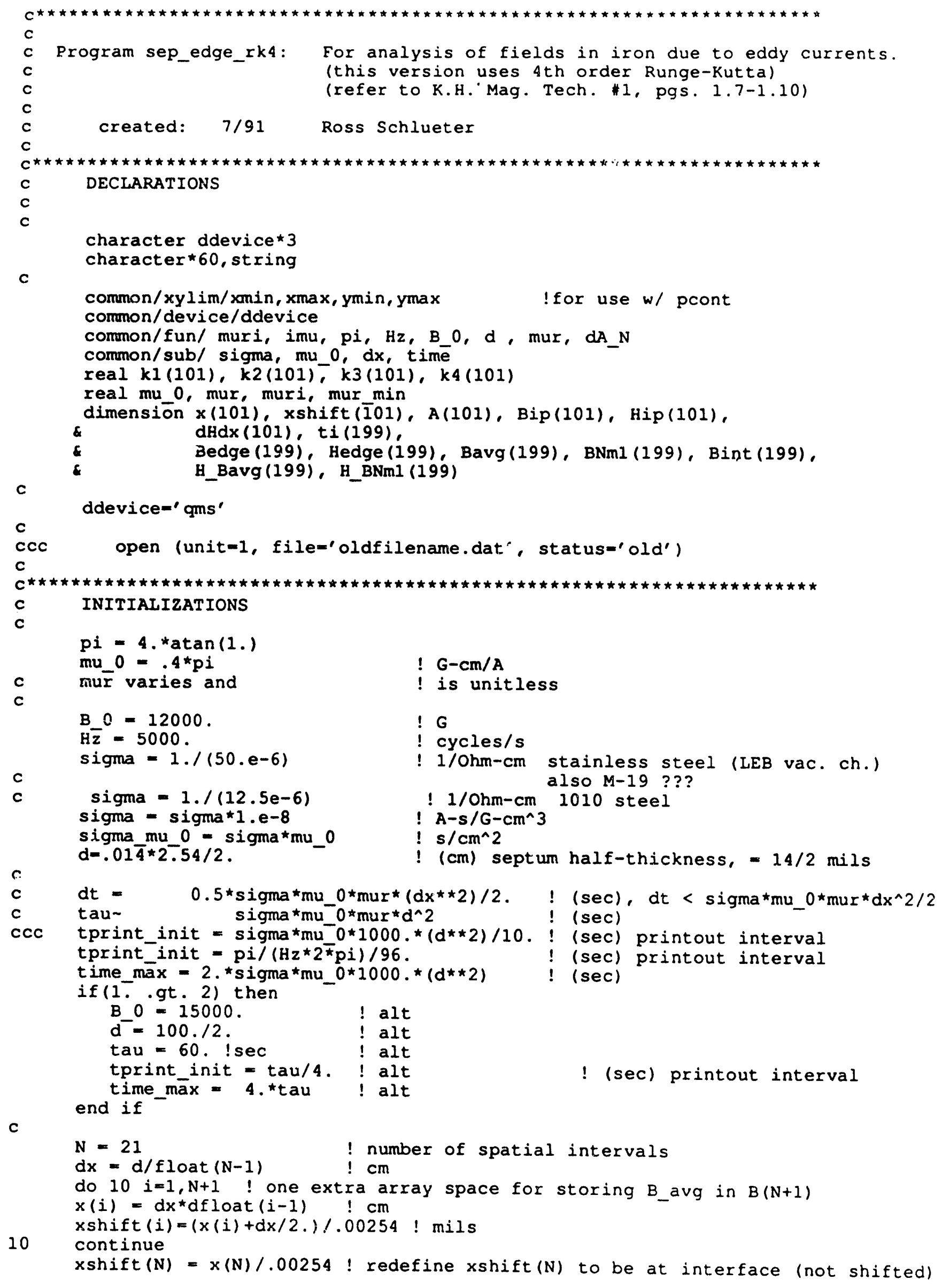




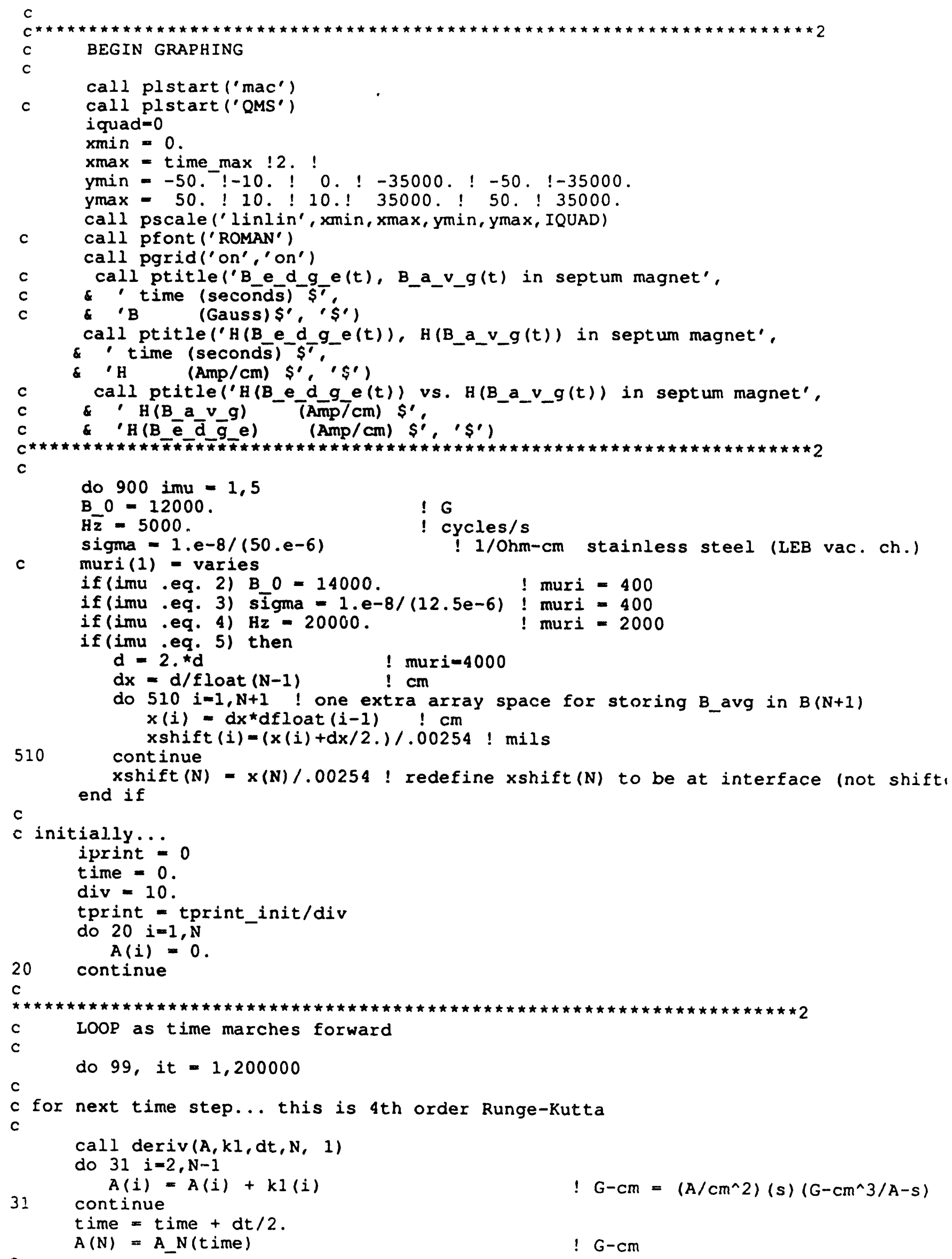




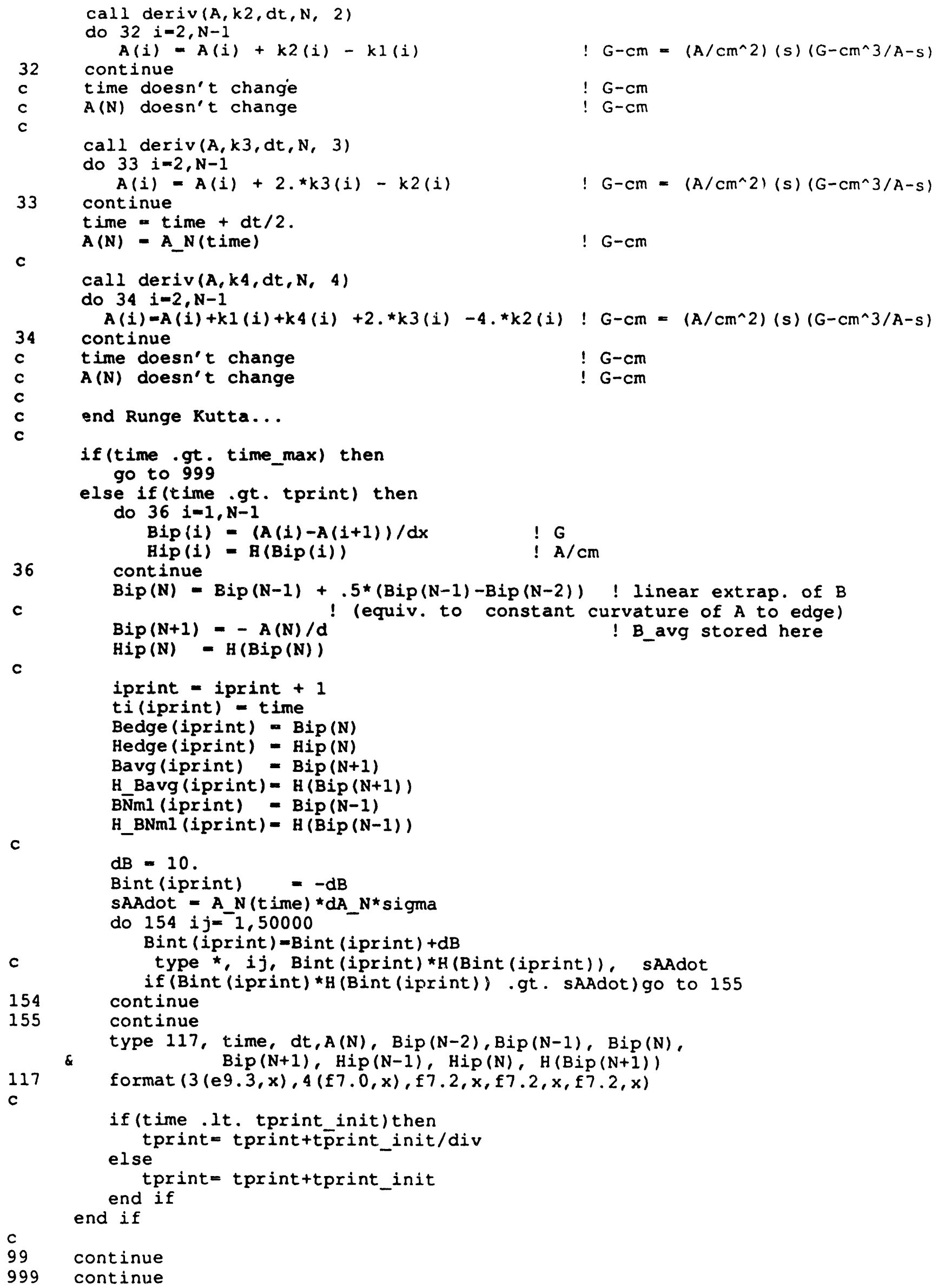




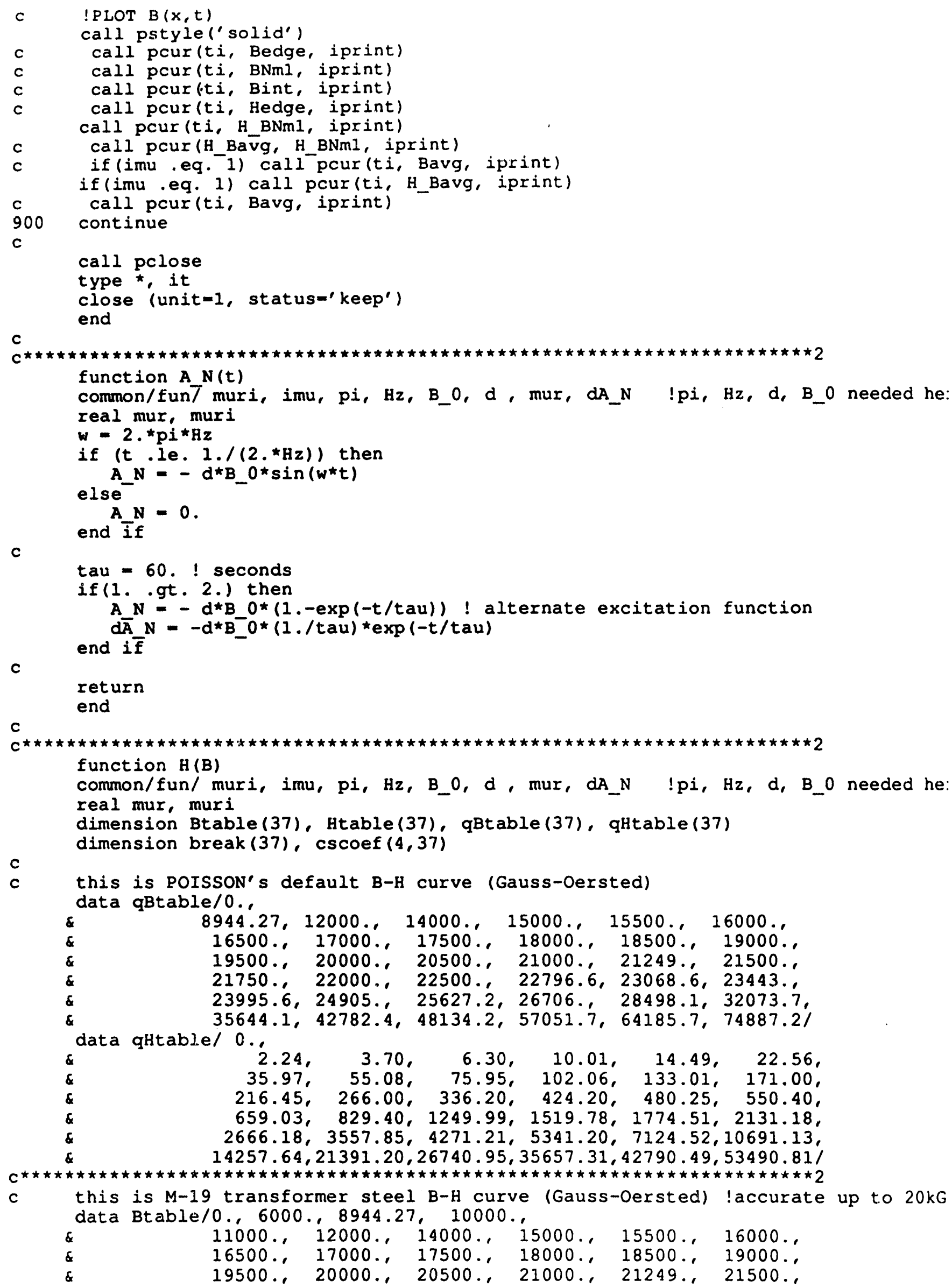




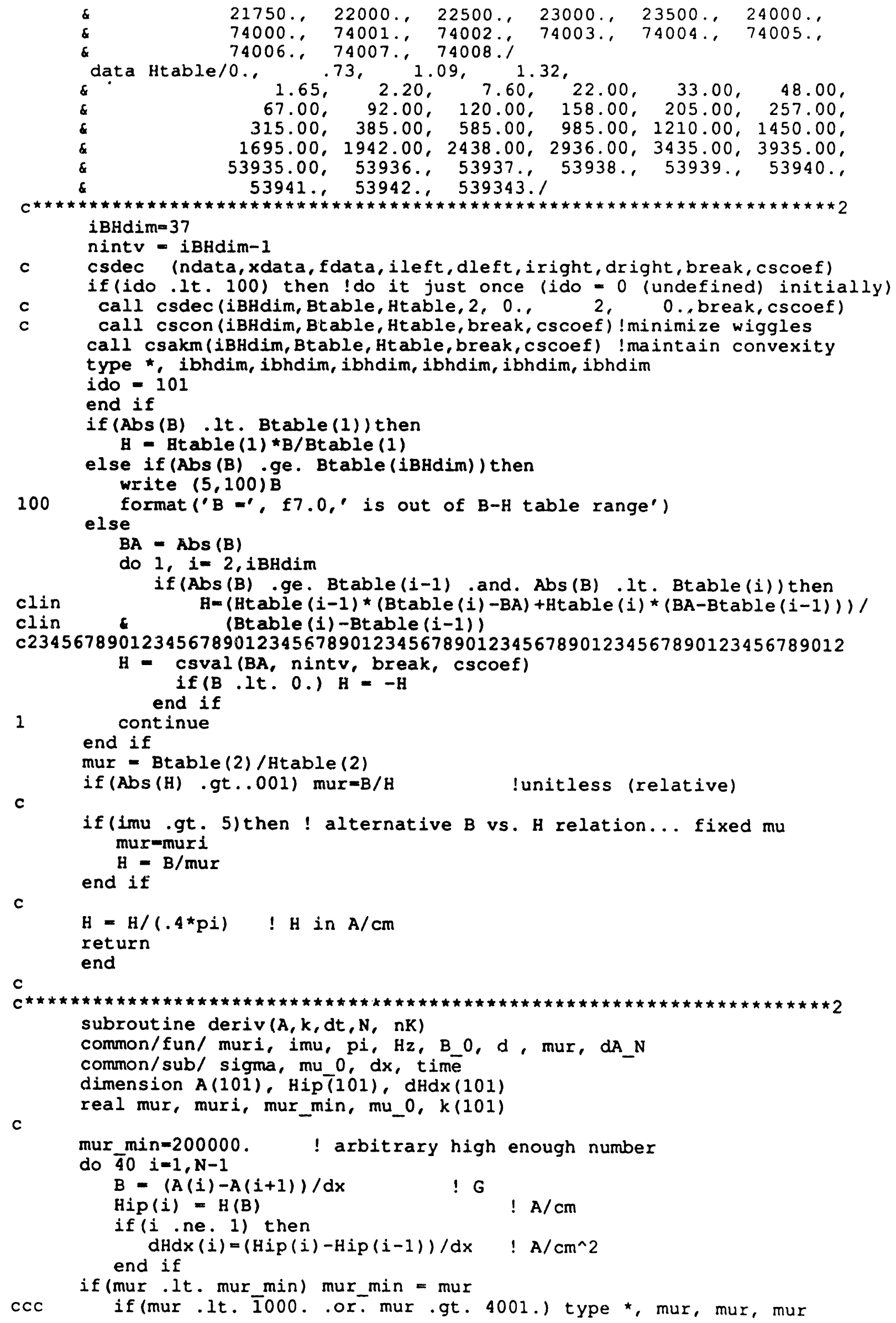




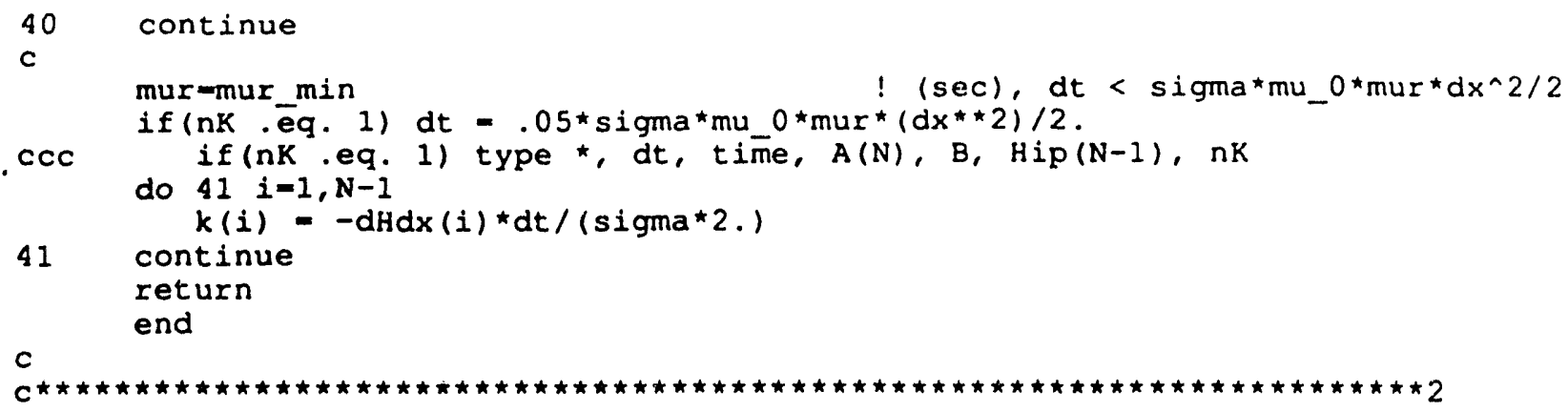



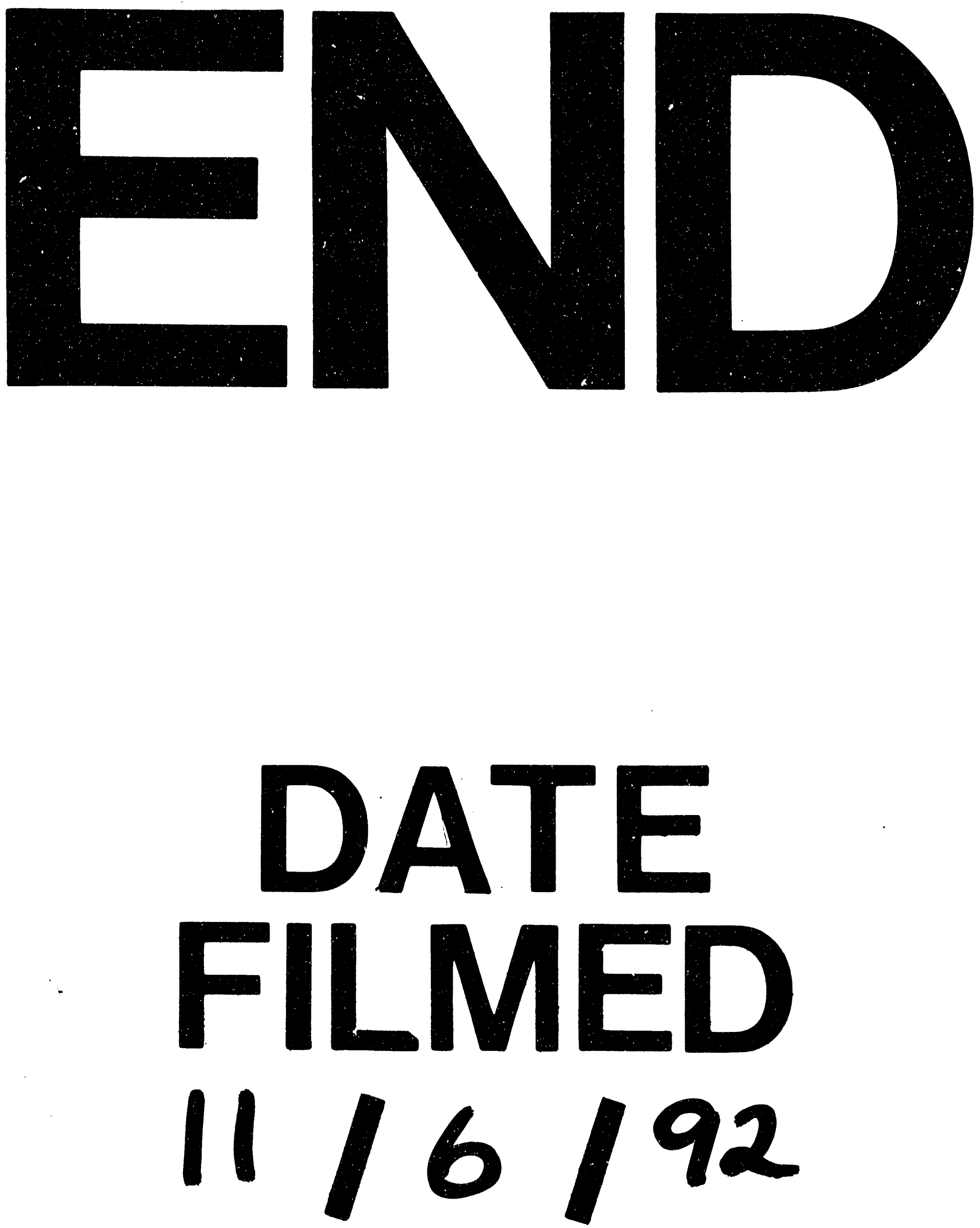

1 
